Disclosure of Interests: None declared

DOI: 10.1136/annrheumdis-2021-eular.330

\section{AB0409 INTRAVENOUS ILOPROST: A REGION WIDE SURVEY OF CURRENT PRESCRIBING AMONGST HOSPITALS IN THE WESSEX MULTI-DISCIPLINARY CONNECTIVE TISSUE DISEASE NETWORK}

L. Parker ${ }^{1}$, A. Mason ${ }^{2}$, B. Davidson ${ }^{2}{ }^{1}$ University Hospitals Dorset NHS Foundation Trust, Rheumatology Department, Christchurch, United Kingdom; ${ }^{2}$ University Hospital Southampton NHS Foundation Trust, Rheumatology Department, Southampton, United Kingdom

Background: Digital ulceration in systemic sclerosis is a severe and disabling aspect of this complex, multi-system disease. Traditionally, intravenous (IV) iloprost has been prescribed to treat the problem. Alternative oral medications including sildenafil and bosentan are now readily available, although NHS clinical commissioning policy is yet to be updated to reflect changes in drug costs. Use of IV iloprost across Wessex was audited to review current practice across the region.

Objectives: To compare use of IV iloprost across 7 different hospital rheumatology departments

Methods: Data was collected across 7 different NHS hospital sites using rheumatology specific pharmacy records for high cost drug prescribing and a single uniform data collection proforma, which was then analysed by 1 person to ensure uniformity of results. Case notes were reviewed retrospectively to determine diagnosis, frequency and duration of iloprost treatment, if patients had been considered for sildenafil treatment (including if sildenafil was prescribed, any cautions or contra-indications to treatment, dose and any reason for discontinuation of treatment) and if patients had been considered for bosentan treatment and if it was prescribed.

Results: 45 patients were identified currently receiving IV iloprost. 32/45 (71\%) had a diagnosis of systemic sclerosis, mixed connective tissue disease (CTD) or undifferentiated CTD (to be called scleroderma group). 13/45 had other miscellaneous diagnoses which had resulted in digital ulceration. These 13 patients were excluded from further analysis relating to sildenafil or bosentan prescribing as these medications were not indicated for other causes of digital ulceration. In the scleroderma group, $27 / 32$ (84\%) had been considered for sildenafil treatment. Of these, 8/27 started and discontinued due to intolerance and 17/27 remained on sildenafil. In the scleroderma group, 9/32 had been considered for bosentan treatment, and of these $7 / 9$ remained on bosentan.

Conclusion: The authors have identified that IV iloprost is still widely used for management of digital ulceration in rheumatology departments across seven different hospitals in Wessex. Most of this group of patients would likely be eligible for treatment with sildenafil or bosentan. Sildenafil intolerance is a challenge to management. Consideration of bosentan as a treatment option was limited in this group, and may be a cost-effective alternative to IV iloprost which would also eliminate the risk of infection associated with IV access in a potentially immunosuppressed group of patients.

Disclosure of Interests: None declared

DOI: 10.1136/annrheumdis-2021-eular.331

\section{AB0410 $100 A 4$ PLASMA LEVELS CORRELATE WITH DISEASE ACTIVITY, SKIN FIBROSIS AND INTERSTITIAL LUNG DISEASE IN SYSTEMIC SCLEROSIS PATIENTS}

H. Štorkánová ${ }^{1,2}$, L. Andres Cerezo ${ }^{1}$, S. Oreska ${ }^{1,2}, M$. Špiritović ${ }^{1,3}$, B. Heřmánková ${ }^{1,3}$, M. Komarc ${ }^{4}$, K. Pavelka ${ }^{1,2}$, J. Vencovský ${ }^{1,2}$, J. H. W. Distler ${ }^{5}$, L. Šenolt ${ }^{1,2}$, R. Bečvárir ${ }^{1,2}, M$. Tomcik ${ }^{1,2}{ }^{1}$ Institute of Rheumatology, -, Prague, Czech Republic; ${ }^{2} 1$ st Faculty of Medicine, Charles University, Prague, Czech Republic, Department of Rheumatology, Prague, Czech Republic; ${ }^{3}$ Faculty of Physical Education and Sport, Charles University, Prague, Czech Republic, Department of Physiotherapy, Prague, Czech Republic; ${ }^{4}$ Faculty of Physical Education and Sport, Charles University, Prague, Czech Republic, Department of Methodology, Prague, Czech Republic; ${ }^{5}$ University of Erlangen-Nuremberg, Erlangen, Germany, Department of Internal Medicine III and Institute for Clinical Immunology, Erlangen, Germany

Background: In our previous study we demonstrated that S100A4 is overexpressed in scleroderma (SSc) skin, SSc fibroblasts and preclinical models of SSc in a TGF- $\beta$ dependent manner. We showed that S100A4 is a new regulator of TGF- $\beta$ signalling and its inhibition prevents the pro-fibrotic effects of TGF- $\beta$. Inactivation of S100A4 prevented dermal fibrosis induced by bleomycin and in Tsk-1 mice

Objectives: The aim of this study was to evaluate S100A4 in the peripheral blood of SSc patients and characterize its potential association with SSc-related features.
Methods: A total of 33 patients (29 females; mean age 52.8; disease duration 4.2 years; dcSSc/lcSSc $=8 / 25$ ) who met the 2013 EULAR/ACR classification criteria for SSc and 20 healthy age- and sex-matched individuals were included in this study. Plasma levels of S100A4 were measured using ELISA (CUSABIO, Houston, USA). Data are presented as median (IQR).

Results: S100A4 plasma levels were significantly increased in SSc patients compared to healthy controls (78.6(32.3-146.5) vs. 43.4(32.3-53.4)ng/ $m L, p=0.011$ ). Patients with diffuse cutaneous (dc)SSc had significantly higher levels of S100A4 than patients with limited cutaneous (Ic)SSc or healthy con trols (168.5(81.5-347.5) vs. 63.4(30.9-130.6), $\mathrm{p}=0.017, \mathrm{p}=0.001$, respectively) Plasma levels of S100A4 positively correlated with mRSS $(r=0.556, p=0.001)$. Furthermore, S100A4 negatively correlated with forced vital capacity (FVC) and peripheral oxygen saturation (SpO2) $(r=-0.362, p=0.038 ; r=-0.414, p=0.029$, respectively). S100A4 levels positively correlated with ESSG activity score $(r=0.750, p<0.001)$. However, only correlations between S100A4 and mRSS, and ESSG activity score were approved at corrected level of statistical sig nificance after Bonferroni's correction $(p<0.01)$. In a prospective analysis of patients $(n=40)$ with progressive SSc-ILD treated with $6(n=24)$ or $12(n=16)$ monthly i.v. pulses of cyclophosphamide (CPA, $500 \mathrm{mg} / \mathrm{m}^{2}$ ), we observed a significant decrease in plasma S100A4 levels between the baseline samples (month 0 ) and blood drawn after 6 months of CPA treatment (76.3(52.9-98.6) vs. 73.2(44.4-98.6) $\mathrm{ng} / \mathrm{mL}, \mathrm{p}=0.013$ ). Furthermore, baseline $\mathrm{S} 100 \mathrm{~A} 4$ levels predicted the change (m0-m6) in CRP and ESR levels after 6 months of CPA therapy $(r=0.472, p=0.004 ; r=0.528, p=0.003$, respectively).

Conclusion: We demonstrate that plasma S100A4 levels are significantly increased in SSc patients compared with healthy controls. Increased S100A4 is associated with the dcSSc subset, skin involvement, deteriorated parameters of interstitial lung disease and higher disease activity. In patients with progressive SSc-ILD, S100A4 declines after 6 months of cyclophosphamide therapy and predicts the systemic inflammatory response. These data further suppor our previous findings on the role of S100A4 as a regulator of TGF- $\beta$ induced fibrosis in SSc.

Acknowledgements: Supported by MHCR023728, SVV-260373.

Disclosure of Interests: None declared

DOI: 10.1136/annrheumdis-2021-eular.462

\section{AB0411 $\quad$ CHALLENGES IN THE MANAGEMENT OF MIXED CONNECTIVE TISSUE DISEASE: A RETROSPECTIVE ANALYSIS OF THE MCTD COHORT IN A TERTIARY REFERRAL CENTRE}

A. Wanzenried ${ }^{1}$, A. Garaiman ${ }^{1}$, S. Jordan ${ }^{1}$, O. Distler ${ }^{1}$, B. Maurer ${ }^{1} .{ }^{1}$ University Hospital Zurich, Department of Rheumatology, Zürich, Switzerland

Background: As a rare, complex, and heterogeneous disease, mixed connective tissue disease (MCTD) represents a challenge for clinical practice.

Objectives: We aimed to unravel potential pitfalls including correct referral diagnosis, fulfilment of diagnostic criteria, distinction from other CTDs, disease course and activity, and treatment modalities.

Methods: We analysed the prospectively collected MCTD cohort at our tertiary referral centre. The patients' medical histories were investigated for fulfilment of Sharp's (1), Kasukawa's (2), and Alarcón-Segovia's (3) diagnostic MCTD criteria. We defined overlap syndromes as simultaneous fulfilment of clinical as well as immunological criteria of two defined rheumatic diseases. Disease conversion was defined as emergence of new symptoms and autoantibodies consistent with another rheumatic disease. Remission was defined by simultaneous systemic lupus erythematosus disease activity index 2000 (SLEDAI-2 K) of 0 and European League Against Rheumatism scleroderma trial and research (EUSTAR) activity index $<2.5$. Disease phenotype and disease activity were monitored over time and all patients were evaluated for fulfilment of classification criteria of various connective tissue diseases.

Results: Out of 85 patients initially referred as MCTD, only one third fulfilled the diagnostic MCTD criteria. Most of the remaining patients had undifferentiated CTD (29\%) or overlap syndromes $(20 \%)$. In our final cohort of 33 MCTD patients, 6 (48\%) also met the classification criteria of systemic sclerosis, $13(39 \%)$ those of systemic lupus erythematosus (SLE), $6(18 \%)$ those of rheumatoid arthritis, and $3(9 \%)$ those of primary myositis. Over the median observation period of $4.6(1.6,9.9)$ years, only two patients $(6 \%)$ underwent disease conversion from MCTD to SLE and no patient converted towards other diseases. The number of patients in remission increased from $6(18 \%)$ to $15(45 \%)$ due to introduction of immune modulatory treatment. Combination therapy was favoured in most cases (17 patients, $52 \%$ ), whereas monotherapy was less frequent (12 patients, 36\%), and only 4 (12\%) patients remained without immune modulators until the end of the follow-up period. Hydroxychloroquine, prednisone, and methotrexate were the most frequently used medications in our cohort. 